International Journal of Pure and Applied Mathematics

Volume 115 No. 1 2017, 67-75

ISSN: 1311-8080 (printed version); ISSN: 1314-3395 (on-line version)

url: http://www.ijpam.eu

doi: 10.12732/ijpam.v115i1.6

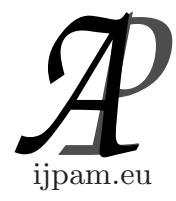

\title{
GENERALIZED VARIABLE PRECISION ROUGH SETS WITH THE INCLUSION ERRORS
}

\author{
Ju-Mok $\mathrm{Oh}^{1}$, Yong Chan $\mathrm{Kim}^{2} \S$ \\ ${ }^{1,2}$ Department of Mathematics \\ Gangneung-Wonju University \\ Gangneung, Gangwondo 25457, KOREA
}

\begin{abstract}
In this paper, we introduce the notion of generalized variable precision rough sets with the inclusion errors. We investigate the properties of generalized upper approximation operators and generalized lower approximation operators with the inclusion errors, respectively. We give their examples.
\end{abstract}

AMS Subject Classification: 03E72, 06A15, 06F07

Key Words: inclusion errors, generalized lower (upper) approximation operators, generalized variable precision rough sets

\section{Introduction}

Pawlak $[7,8]$ introduced the rough set theory as a formal tool to deal with imprecision and uncertainty in the data analysis. It is based on equivalence relation and crisp sets. Dudois and Prade [4] introduced the notions of fuzzy rough sets as fuzziness of concepts and vagueness of information in decision making. The relationship between rough set theory and topological spaces was investigated in sets [6,16]. As an extension of rough sets, Ziarko [17] introduced the variable precision rough set which cannot only solve problems with uncertain data, but also relax the strict definition of the rough set. He studied the relative

Received: $\quad$ February 7, 2017

Revised: $\quad$ May 2, 2017

Published: $\quad$ June 28, 2017

(c) 2017 Academic Publications, Ltd. url: www.acadpubl.eu

$\S_{\text {Correspondence author }}$ 
error limit of the partition blocks with the inclusion order $A \subset_{\beta} B$ iff $e(A, B) \leq$ $\beta$.

Formal concept analysis [1] and rough set theory are two important mathematical tools for data analysis and knowledge processing. A formal concept consists of $(X, Y, R)$ where $X$ is a set of objects, $Y$ is a set of attributes and $R$ is a relation between $X$ and $Y$. Classical algebraic structures [11-15] in the rough set theory are considered as two types as follows: for $A \subset X$ and a relation $R \subset$ over universe $X$ and $R[x]=\{y \in X \mid(x, y) \in R\}$,

$$
\begin{aligned}
& \underline{A}=\{x \in X \mid R[x] \subset A\}, \\
& \bar{A}=\{x \in X \mid R[x] \cap A \neq \emptyset\} .
\end{aligned}
$$

The pair $(\underline{A}, \bar{A})$ is called a generalized rough set of $A$. As an extension of Ziarko's rough sets, Dai et al.[3] introduced the generalized variable precision rough set $(\underline{A}, \bar{A})$ defined by

$$
\begin{aligned}
& \operatorname{apr}_{R}^{\beta}(A)=\bigcup\left\{R[x] \mid x \in R^{-1}[y], e\left(R^{-1}[y], A\right) \leq \beta\right\}, \\
& \overline{a p r}_{R}^{\beta}(A)=\bigcup\left\{R[x] \mid x \in R^{-1}[y], e\left(R^{-1}[y], A\right)<1-\beta\right\} .
\end{aligned}
$$

In this paper, we introduce the notion of generalized variable precision rough sets with the inclusion errors as an extension of [3,17]. We investigate the properties of generalized upper approximation operator $\underline{R}^{\beta}$ and generalized lower approximation operator $\bar{R}^{\beta}$ with $e_{L^{Y}}$ and $d_{L^{Y}}$, respectively. We give their examples.

\section{Preliminaries}

Definition 2.1. $[3,17]$ Let $P(X)=\{A \mid A \subset X\}$ be given. A map $e: P(X) \times P(X) \rightarrow[0,1]$ is called an inclusion error defined by

$$
e(A, B)=\left\{\begin{array}{l}
1-\frac{n(A \cap B)}{n(A)}, \quad A \neq \emptyset \\
0, \quad A=\emptyset
\end{array}\right.
$$

where $n(A)$ is the number of elements in $A$.

Definition 2.2. [3,17] Let $X$ and $Y$ be sets, $R \subset X \times Y$. For $\beta \in[0,0.5)$ and $A \subset B$,

(1) ${ }_{\operatorname{apr}}^{\beta}: P(X) \rightarrow P(Y)$ is called a $\beta$-lower approximation defined by

$$
\operatorname{apr}_{R}^{\beta}(A)=\bigcup\left\{R[x] \mid x \in R^{-1}[y], e\left(R^{-1}[y], A\right) \leq \beta\right\} .
$$


(2) $\overline{a p r}_{R}^{\beta}: P(X) \rightarrow P(Y)$ is called a $\beta$-upper approximation defined by

$$
\overline{\operatorname{apr}}_{R}^{\beta}(A)=\bigcup\left\{R[x] \mid x \in R^{-1}[y], e\left(R^{-1}[y], A\right)<1-\beta\right\} .
$$

(3) bnd $_{R}^{\beta}: P(X) \rightarrow P(Y)$ is called a $\beta$-boundary region defined by

$$
\operatorname{bnd}_{R}^{\beta}(A)=\bigcup\left\{R[x] \mid x \in R^{-1}[y], \beta<e\left(R^{-1}[y], A\right)<1-\beta\right\} .
$$

(4) $\operatorname{neg}_{R}^{\beta}: P(X) \rightarrow P(Y)$ is called a $\beta$-negative region defined by

$$
n e g_{R}^{\beta}(A)=\bigcup\left\{R[x] \mid x \in R^{-1}[y], e\left(R^{-1}[y], A\right) \geq 1-\beta\right\} .
$$

\section{Generalized Granular Variable Precision Approximation Operators}

Definition 3.1. Let $R \subset X \times Y$ be a relation and $\beta \in[0,1]$.

(1) Two maps $\underline{R}^{\beta}, \bar{R}^{\beta}: P(Y) \rightarrow P(Y)$ are defined as follows: for all $B \in$ $P(Y)$,

$$
\begin{aligned}
& \underline{R}^{\beta}(B)=\bigcup\left\{R[x] \mid e_{P(Y)}(R[x], B) \geq \beta\right\} \\
& \bar{R}^{\beta}(B)=\bigcap\left\{R^{c}[x] \mid d_{P(Y)}\left(R^{c}[x], B\right) \leq \beta\right\}
\end{aligned}
$$

where $e_{P(Y)}, d_{P(Y)}: P(Y) \times P(Y) \rightarrow[0,1]$ as

$$
\begin{aligned}
& e_{P(Y)}(A, B)=\frac{n\left(A^{c} \cup B\right)}{n(Y)} \\
& d_{P(Y)}(A, B)=\frac{n\left(A^{c} \cap B\right)}{n(Y)}
\end{aligned}
$$

where $n(A)$ is the number of elements in $A$.

Then $\underline{R}^{\beta}$ and $\bar{R}^{\beta}$ are called the generalized lower approximation operator on $Y$ and generalized upper approximation operator on $Y$, respectively. The pair $\left(\underline{R}^{\beta}, \bar{R}^{\beta}\right)$ is called the generalized variable precision rough set determined by $e_{P(Y)}$ and $d_{P(Y)}$.

(2) Two maps $\underline{R}^{-1}{ }^{\beta}, \bar{R}^{-1} \beta: P(X) \rightarrow P(X)$ are defined as follows: for all $A \in P(X)$,

$$
\begin{aligned}
& \frac{R^{-1} \beta}{\overline{R^{-1}}} \beta(A)=\bigcup\left\{R^{-1}[y] \mid e_{P(X)}\left(R^{-1}[y], A\right) \geq \beta\right\} \\
& =\bigcap\left\{R^{-1 c}[y] \mid d_{P(X)}\left(R^{-1 c}[y], A\right) \leq \beta\right\}
\end{aligned}
$$


Then $\underline{R}^{-1} \beta$ and $\bar{R}^{-1}$ are called the generalized lower approximation operator on $X$ and generalized upper approximation operator on $X$, respectively. The pair $\left(\underline{R}^{-1}{ }^{\beta}, \bar{R}^{-1}{ }^{\beta}\right)$ is the generalized variable precision rough set determined by $e_{P(X)}$ and $d_{P(X)}$.

Remark 3.2. (1) If $\beta=1$, since $e_{P(Y)}(R[x], B)=1$, then $R[x]^{c} \cup B=Y$;i.e. $R[x] \subset B$. Hence

$$
\underline{R}^{1}(B)=\bigcup\{R[x] \mid R[x] \subset B\} .
$$

If $\beta=0$, since $d_{P(Y)}\left(R^{c}[x], B\right)=0, R[x] \cup B=\emptyset$. Then $B \subset R^{c}[x]$. Hence

$$
\bar{R}^{0}(B)=\bigcap\left\{R^{c}[x] \mid B \subset R^{c}[x]\right\} .
$$

Theorem 3.3. Let $R \subset X \times Y$ be a relation and $\beta \in[0,1]$.

(1) $d_{P(Y)}\left(R^{c}[x], B^{c}\right)=1-e_{P(Y)}(R[x], B)$ for each $B \in P(Y)$.

(2) $\underline{R}^{\beta}(B)=\left(\bar{R}^{1-\beta}\left(B^{c}\right)\right)^{c}$ for each $B \in P(Y)$.

(3) $\underline{R}^{1}(\emptyset)=\emptyset$ and $\bar{R}^{0}(Y)=Y$.

(4) $\underline{R}^{1}(Y)=\bigcup_{x \in X} R[x]$ and $\bar{R}^{0}(\emptyset)=\bigcap_{x \in X} R^{c}[x]$.

(5) If for each $y \in Y$, there exists $x \in X$ such that $y \in R[x]$, then $\underline{R}^{1}(Y)=Y$ and $\bar{R}^{0}(\emptyset)=\emptyset$.

Proof (1) It follows from:

$$
\begin{aligned}
& d_{P(Y)}\left(R^{c}[x], B^{c}\right)=\frac{n\left(R[x] \cap B^{c}\right)}{n(Y)} \\
& =\frac{n(Y)-n\left(R^{c}[x] \cup B\right)}{n(Y)}=1-e_{P(Y)}(R[x], B) .
\end{aligned}
$$

(2)

$$
\begin{aligned}
& \left(\bar{R}^{1-\beta}\left(B^{c}\right)\right)^{c}=\left(\bigcap\left\{R^{c}[x] \mid d_{P(Y)}\left(R^{c}[x], B\right) \leq \beta\right\}\right)^{c} \\
& =\bigcup\left\{R[x] \mid d_{P(Y)}\left(R^{c}[x], B^{c}\right) \leq 1-\beta\right\} \\
& =\bigcup\left\{R[x] \mid 1-e_{P(Y)}(R[x], B) \leq 1-\beta\right\} \\
& =\underline{R}^{\beta}(B) .
\end{aligned}
$$

$$
\begin{aligned}
& \underline{R}^{1}(Y)=\bigcup\{R[x] \mid R[x] \subset Y\}=\bigcup_{x \in X} R[x] \\
& \bar{R}^{0}(\emptyset)=\bigcap\left\{R^{c}[x] \mid \emptyset \subset R^{c}[x]\right\}=\bigcap_{x \in X} R^{c}[x] .
\end{aligned}
$$

(5) Since for each $y \in Y$, there exists $x \in X$ such that $y \in R[x]$, we have $Y=\bigcup_{x \in X} R[x]$. Hence $\underline{R}^{1}(Y)=Y$ and $\bar{R}^{0}(\emptyset)=\emptyset$. 
Corollary 3.4. Let $R^{-1} \subset Y \times X$ be a relation and $\beta \in[0,1]$.

(1) $d_{P(X)}\left(R^{-1 c}[y], A^{c}\right)=1-e_{P(X)}\left(R^{c}[y], A\right)$ for each $A \in P(X)$.

(2) $\underline{R}^{-1} \beta(A)=\left(\bar{R}^{-1}{ }^{1-\beta}\left(A^{c}\right)\right)^{c}$ for each $A \in P(X)$.

(3) $\underline{R}^{-1} 1(\emptyset)=\emptyset$ and ${\overline{R^{-1}}}^{0}(X)=X$.

(4) $\underline{R}^{-1} 1(X)=\bigcup_{y \in Y} R^{-1}[y]$ and ${\overline{R^{-1}}}^{0}(\emptyset)=\bigcap_{y \in Y} R^{-1 c}[y]$.

(5) If for each $x \in X$, there exists $y \in Y$ such that $x \in R^{-1}[y]$, then $\underline{R}^{-1} 1(X)=X$ and ${\overline{R^{-1}}}^{0}(\emptyset)=\emptyset$.

Theorem 3.5. Let $R \subset X \times Y$ be a relation and $\beta \in[0,1]$.

(1) $\underline{R}^{1}(B) \subset B$ and $B \subset \bar{R}^{0}(B)$

(2) If $\beta_{1} \leq \beta_{2}$, then $\underline{R}^{\beta_{2}}(B) \subset \underline{R}^{\beta_{1}}(B)$.

(3) If $B_{1} \subset B_{2}$, then $\underline{R}^{\beta}\left(B_{1}\right) \subset \underline{R}^{\beta}\left(B_{2}\right)$.

(4) If $\beta_{1} \leq \beta_{2}$, then $\bar{R}^{\beta_{2}}(B) \subset \bar{R}^{\beta_{1}}(B)$.

(5) If $B_{1} \subset B_{2}$, then $\bar{R}^{\beta}\left(B_{1}\right) \subset \bar{R}^{\beta}\left(B_{2}\right)$.

(6) $\underline{R}^{1}(R[x])=R[x]$ and $\bar{R}^{0}\left(R^{c}[x]\right)=R^{c}[x]$.

(7) $\underline{R}^{1}\left(\underline{R}^{\beta}(B)\right)=\underline{R}^{\beta}(B)$ and $\bar{R}^{0}\left(\bar{R}^{\beta}(B)\right)=\bar{R}^{\beta}(B)$.

Proof (1) By Remark 3.2, we have $\underline{R}^{1}(B)=\bigcup\{R[x] \mid R[x] \subset B\} \subset B$. Moreover, $\bar{R}^{0}(B)=\bigcap\left\{R^{c}[x] \mid B \subset R^{c}[x]\right\} \supset B$.

$(2),(3)$ and (4) are easily proved.

(6) Since $e_{P(Y)}(R[x], R[x])=1, \underline{R}^{1}(R[x]) \supset R[x]$. By $(1), \underline{R}^{1}(R[x]) \subset R[x]$. Hence $\underline{R}^{1}(R[x])=R[x]$.

Since $d_{P(Y)}\left(R^{c}[x], R^{c}[x]\right)=0, \bar{R}^{0}\left(R^{c}[x]\right) \subset R^{c}[x] . \quad$ By $(1), \bar{R}^{0}\left(R^{c}[x]\right) \supset$ $R^{c}[x]$. Hence $\bar{R}^{0}\left(R^{c}[x]\right)=R^{c}[x]$.

(7) For $R[x] \subset \underline{R}^{\beta}(B), e_{P(Y)}\left(R[x], \underline{R}^{\beta}(B)\right)=1, \underline{R}^{1}\left(\underline{R}^{\beta}(B)\right) \supset \underline{R}^{\beta}(B)$. Вy (1), $\underline{R}^{1}\left(\underline{R}^{\beta}(B)\right) \subset \underline{R}^{\beta}(B)$. Hence $\underline{R}^{1}\left(\underline{R}^{\beta}(B)\right)=\underline{R}^{\beta}(B)$.

For $R^{c}[x] \supset \bar{R}^{\beta}(B), d_{P(Y)}\left(R^{c}[x], \bar{R}^{\beta}(B)\right)=0, \bar{R}^{0}\left(\bar{R}^{\beta}(B)\right) \subset \bar{R}^{\beta}(B)$. Вy (1), $\bar{R}^{1}\left(\bar{R}^{\beta}(B)\right) \supset \bar{R}^{\beta}(B)$. Hence $\bar{R}^{1}\left(\bar{R}^{\beta}(B)\right)=\bar{R}^{\beta}(B)$.

Corollary 3.6. Let $R \subset X \times Y$ be a relation and $\beta \in[0,1]$.

(1) (1) $\underline{R}^{-1}(A) \subset A$ and $A \subset{\overline{R^{-1}}}^{0}(A)$ for each $A \in P(X)$.

(2) If $\beta_{1} \leq \beta_{2}$, then $\underline{R}^{-1 \beta_{2}}(A) \subset \underline{R}^{-\beta_{1}}(A)$.

(3) If $A_{1} \subset A_{2}$, then $\underline{R^{-1} \beta}\left(A_{1}\right) \subset \underline{R^{-1}} \beta\left(A_{2}\right)$.

(4) If $\beta_{1} \leq \beta_{2}$, then $\overline{R^{-1}} \beta_{2}(A) \subset \overline{R^{-1}} \beta_{1}(A)$.

(5) If $A_{1} \subset A_{2}$, then $\bar{R}^{\beta}{ }^{\beta}\left(A_{1}\right) \subset{\overline{R^{-1}}}^{\beta}\left(A_{2}\right)$.

(6) $\underline{R}^{-1}(R[y])=R^{-1}[y]$ and ${\overline{R^{-1}}}^{0}\left(R^{-1 c}[y]\right)=R^{-1 c}[y]$. 
(7) $\underline{R}^{-1}\left(\underline{R}^{-1}(A)\right)=\underline{R}^{-1} \beta(A)$ and ${\overline{R^{-1}}}^{0}\left({\overline{R^{-1}}}^{\beta}(A)\right)={\overline{R^{-1}}}^{\beta}(A)$.

Theorem 3.7. Let $R \subset X \times Y$ be a relation and $\beta \in[0,1]$. Define $\mathcal{T}_{Y}, \mathcal{F}_{Y} \subset P(Y)$ as

$$
\begin{aligned}
& \mathcal{T}_{Y}=\left\{B \in P(Y) \mid B=\underline{R}^{1}(B)\right\}, \\
& \mathcal{F}_{Y}=\left\{B \in P(Y) \mid B=\bar{R}^{0}(B)\right\} .
\end{aligned}
$$

(1) $\emptyset \in \mathcal{T}_{Y}, R[x] \in \mathcal{T}_{Y}$ and and $\underline{R}^{\beta}(B) \in \mathcal{T}_{Y}$ for each $B \in P(Y), \beta \in[0,1]$.

(2) If $B_{i} \in \mathcal{T}_{Y}$ for each $i \in \Gamma$, then $\bigcup_{i \in \Gamma} B_{i} \in \mathcal{T}_{Y}$.

(3) $Y \in \mathcal{F}_{Y}, R^{c}[x] \in \mathcal{F}_{Y}$ and $\bar{R}^{\beta}(B) \in \mathcal{F}_{X}$ for each $B \in P(Y), \beta \in[0,1]$.

(4) If $B_{i} \in \mathcal{F}_{Y}$ for each $i \in \Gamma$, then $\bigcap_{i \in \Gamma} B_{i} \in \mathcal{F}_{Y}$.

(5) $B \in \mathcal{T}_{Y}$ iff $B^{c} \in \mathcal{F}_{Y}$.

(6) If for each $y \in Y$, there exists $x \in X$ such that $y \in R[x]$, then $Y \in \mathcal{T}_{Y}$ and $\emptyset \in \mathcal{F}_{Y}$.

Proof (1) By Theorems 3.3(3) and 3.5(6,7), we have $\emptyset=\underline{R}^{1}(\emptyset), R[x]=$ $\underline{R}^{1}(R[x])$ and $\underline{R}^{\beta}(B)=\underline{R}^{1}\left(\underline{R}^{\beta}(B)\right)$ for each $B \in P(Y), \beta \in[0,1]$.

(2) Let $B_{i} \in \mathcal{T}_{Y}$ for each $i \in \Gamma$. By Theorem 3.5(1,3), since $\bigcup_{i \in I} B_{i}=$ $\bigcup_{i \in I} \underline{R}^{1}\left(B_{i}\right) \subset \underline{R}^{1}\left(\bigcup_{i \in I} B_{i}\right) \subset \bigcup_{i \in I} B_{i}$, we have $\bigcup_{i \in I} B_{i} \in \mathcal{T}_{Y}$.

(3) By Theorems 3.3(3) and $3.5(6,7)$, we have $Y=\bar{R}^{0}(Y), R^{c}[x]=$ $\bar{R}^{0}\left(R^{c}[x]\right)$ and $\bar{R}^{\beta}(B)=\bar{R}^{0}\left(\bar{R}^{\beta}(B)\right)$ for each $B \in P(Y), \beta \in[0,1]$.

(4) Let $B_{i} \in \mathcal{F}_{Y}$ for each $i \in \Gamma$. By Theorem 3.5(1,5), since $\bigcap_{i \in I} B_{i}=$ $\bigcap_{i \in I} \bar{R}^{0}\left(B_{i}\right) \supset \bar{R}^{1}\left(\bigcap_{i \in I} B_{i}\right) \supset \bigcap_{i \in I} B_{i}$, we have $\bigcap_{i \in I} B_{i} \in \mathcal{F}_{Y}$.

(5) By Theorem $3.3(1), B=\underline{R}^{1}(B)=\left(\bar{R}^{0}\left(B^{c}\right)\right)^{c}$ iff $B^{c}=\bar{R}^{0}\left(B^{c}\right)$.

(6) It follows from Theorem 3.5(6).

Corollary 3.8. Let $R \subset X \times Y$ be a relation and $\beta \in[0,1]$. Define $\mathcal{T}_{X}, \mathcal{F}_{X} \subset P(X)$ as

$$
\begin{aligned}
& \mathcal{T}_{X}=\left\{A \in P(X) \mid A={\underline{R^{-1}}}^{1}(A)\right\}, \\
& \mathcal{F}_{X}=\left\{A \in P(X) \mid A={\overline{R^{-1}}}^{0}(A)\right\} .
\end{aligned}
$$

(1) $\emptyset \in \mathcal{T}_{X}, R^{-1}[x] \in \mathcal{T}_{X}$ and $\underline{R^{-1} \beta}(A) \in \mathcal{T}_{X}$ for each $A \in P(X), \beta \in[0,1]$.

(2) If $A_{i} \in \mathcal{T}_{X}$ for each $i \in \Gamma$, then $\bigcup_{i \in \Gamma} A_{i} \in \mathcal{T}_{X}$.

(3) $X \in \mathcal{F}_{X}, R^{-1 c}[x] \in \mathcal{F}_{X}$ and ${\overline{R^{-1}}}^{\beta}(A) \in \mathcal{T}_{X}$ for each $A \in P(X), \beta \in$ $[0,1]$.

(4) If $A_{i} \in \mathcal{F}_{X}$ for each $i \in \Gamma$, then $\bigcap_{i \in \Gamma} A_{i} \in \mathcal{F}_{X}$.

(5) $A \in \mathcal{T}_{X}$ iff $A^{c} \in \mathcal{F}_{X}$. 
(6) If for each $x \in X$, there exists $y \in Y$ such that $x \in R^{-1}[y]$, then $X \in \mathcal{T}_{X}$ and $\emptyset \in \mathcal{F}_{X}$.

Example 3.9. Let $X=\left\{x_{1}, x_{2}, x_{3}, x_{4}, x_{5}, x_{6}\right\}, Y=\left\{y_{1}, y_{2}, y_{3}, y_{4}, y_{5}\right\}$ and $R \subset X \times Y$ as follows

$$
\begin{gathered}
R=\left\{\left(x_{1}, y_{1}\right),\left(x_{1}, y_{2}\right),\left(x_{1}, y_{4}\right),\left(x_{2}, y_{1}\right),\left(x_{2}, y_{2}\right),\left(x_{2}, y_{3}\right),\left(x_{3}, y_{2}\right),\left(x_{3}, y_{4}\right),\right. \\
\left.\left(x_{3}, y_{5}\right),\left(x_{4}, y_{4}\right),\left(x_{4}, y_{5}\right),\left(x_{5}, y_{3}\right),\left(x_{5}, y_{5}\right),\left(x_{6}, y_{3}\right)\right\} .
\end{gathered}
$$

We obtain the followings:

$$
\begin{aligned}
R\left[x_{1}\right] & =\left\{y_{1}, y_{2}, y_{4}\right\}, \quad R\left[x_{2}\right]=\left\{y_{1}, y_{2}, y_{3}\right\} \\
R\left[x_{3}\right] & =\left\{y_{2}, y_{4}, y_{5}\right\}, \quad R\left[x_{4}\right]=\left\{y_{4}, y_{5}\right\} \\
R\left[x_{5}\right] & =\left\{y_{3}, y_{5}\right\}, \quad R\left[x_{6}\right]=\left\{y_{3}\right\} \\
R^{c}\left[x_{1}\right] & =\left\{y_{3}, y_{5}\right\}, \quad R^{c}\left[x_{2}\right]=\left\{y_{4}, y_{5}\right\} \\
R^{c}\left[x_{3}\right] & =\left\{y_{1}, y_{3}\right\}, \quad R^{c}\left[x_{4}\right]=\left\{y_{1}, y_{2}, y_{3}\right\} \\
R^{c}\left[x_{5}\right] & =\left\{y_{1}, y_{2}, y_{4}\right\}, \quad R^{c}\left[x_{6}\right]=\left\{y_{1}, y_{2}, y_{4}, y_{5}\right\} \\
R^{-1}\left[y_{1}\right] & =\left\{x_{1}, x_{2}\right\}, \quad R^{-1}\left[y_{2}\right]=\left\{x_{1}, x_{2}, x_{3}\right\} \\
R^{-1}\left[y_{3}\right] & =\left\{x_{2}, x_{5}, x_{6}\right\}, \quad R^{-1}\left[y_{4}\right]=\left\{x_{1}, x_{3}, x_{4}\right\} \\
R^{-1}\left[x_{5}\right] & =\left\{x_{3}, x_{4}, x_{5}\right\}, \quad \\
R^{-1 c}\left[y_{1}\right] & =\left\{x_{3}, x_{4}, x_{5}, x_{6}\right\}, \quad R^{-1 c}\left[y_{2}\right]=\left\{x_{4}, x_{5}, x_{6}\right\} \\
R^{-1 c}\left[y_{3}\right] & =\left\{x_{1}, x_{3}, x_{4}\right\}, \quad R^{-1 c}\left[y_{4}\right]=\left\{x_{2}, x_{5}, x_{6}\right\} \\
R^{-1 c}\left[y_{5}\right] & =\left\{x_{1}, x_{2}, x_{6}\right\} .
\end{aligned}
$$

(1) For $B=\left\{y_{1}, y_{3}, y_{4}\right\}$ and $\beta=0.8$, we obtain $e_{P(Y)}\left(R\left[x_{i}\right], B\right) \geq 0.8$ for $i=1,2,4,5,6$. Thus

$$
\underline{R}^{0.8}(B)=R\left[x_{1}\right] \cup R\left[x_{2}\right] \cup R\left[x_{4}\right] \cup R\left[x_{5}\right] \cup R\left[x_{6}\right]=Y .
$$

For $B^{c}=\left\{y_{2}, y_{5}\right\}$ and $\beta=0.2$, we obtain $d_{P(Y)}\left(R^{c}\left[x_{i}\right], B^{c}\right) \geq 0.8$ for $i=$ $1,2,3,4,5$. Thus

$$
\bar{R}^{0.2}\left(B^{c}\right)=R^{c}\left[x_{1}\right] \cup R^{c}\left[x_{2}\right] \cap R^{c}\left[x_{3}\right] \cap R^{c}\left[x_{4}\right] \cap R^{c}\left[x_{5}\right]=\emptyset .
$$

Hence $\underline{R}^{0.8}(B)=\left(\bar{R}^{0.2}\left(B^{c}\right)\right)^{c}$. Moreover, $\underline{R}^{1}\left(\underline{R}^{0.8}(B)\right)=\underline{R}^{0.8}(B)=Y$ and $\bar{R}^{0}\left(\bar{R}^{0.2}\left(B^{c}\right)\right)=\bar{R}^{0.2}\left(B^{c}\right)=\emptyset$.

(2) For $B=\left\{y_{1}, y_{3}, y_{4}\right\}$ and $\beta=0.2$, we obtain $d_{P(Y)}\left(R^{c}\left[x_{i}\right], B\right) \leq 0.2$ for $i=3,4,5,6$. Thus

$$
\bar{R}^{0.2}(B)=R^{c}\left[x_{3}\right] \cap R^{c}\left[x_{4}\right] \cap R^{c}\left[x_{5}\right] \cap R^{c}\left[x_{6}\right]=\left\{y_{1}\right\} .
$$


For $B^{c}=\left\{y_{2}, y_{5}\right\}$ and $\beta=0.8$, we obtain $e_{P(Y)}\left(R\left[x_{i}\right], B^{c}\right) \geq 0.8$ for $i=$ $3,4,5,6$. Thus

$$
\underline{R}^{0.8}(B)=R\left[x_{3}\right] \cup R\left[x_{4}\right] \cup R\left[x_{5}\right] \cup R\left[x_{6}\right]=\left\{y_{2}, y_{3}, y_{4}, y_{5}\right\} .
$$

Hence $\underline{R}^{0.8}\left(B^{c}\right)=\left(\bar{R}^{0.2}(B)\right)^{c}$. Moreover, $\underline{R}^{1}\left(\underline{R}^{0.8}\left(B^{c}\right)\right)=\underline{R}^{0.8}\left(B^{c}\right)=\left\{y_{2}, y_{3}, y_{4}\right.$, $\left.y_{5}\right\}$ and $\bar{R}^{0}\left(\bar{R}^{0.2}(B)\right)=\bar{R}^{0.2}(B)=\left\{y_{1}\right\}$.

(3) For $A=\left\{x_{3}, x_{4}\right\}$ and $\beta=0.7$, we obtain $e_{P(X)}\left(R^{-1}\left[y_{i}\right], A\right) \geq 0.7$ for $i=4,5$. Thus

$$
\underline{R}^{-1^{0.7}}(A)=R^{-1}\left[y_{4}\right] \cup R^{-1}\left[y_{5}\right]=\left\{x_{1}, x_{3}, x_{4}, x_{5}\right\}
$$

Then $\left.\underline{R}^{-1}{ }^{R^{-1^{0.7}}}(A)\right)=\underline{R}^{-1^{0.7}}(A)=\left\{x_{1}, x_{3}, x_{4}, x_{5}\right\}$.

(4) For $A=\left\{x_{3}, x_{4}\right\}$ and $\beta=0.3$, we obtain $d_{P(X)}\left(R^{-1 c}\left[y_{i}\right], A\right) \leq 0.3$ for $i=1,2,3$. Thus

$$
{\overline{R^{-1}}}^{0.3}(A)=R^{-1 c}\left[y_{1}\right] \cap R^{-1 c}\left[y_{2}\right] \cap R^{-1 c}\left[y_{3}\right]=\left\{x_{2}\right\}
$$

Then ${\overline{R^{-1}}}^{0}\left(\bar{R}^{-1}{ }^{0.3}(A)\right)={\overline{R^{-1}}}^{0 . .3}(A)=\left\{y_{4}\right\}$.

(5) Since $R$ satisfies the conditions of Theorem 3.3(5) and Corollary 3.4(5), $\underline{R}^{1}(Y)=Y, \bar{R}^{0}(\emptyset)=\emptyset, \underline{R}^{-1}(X)=X$ and $\bar{R}^{-1}(\emptyset)=\emptyset$

(6) By (5), Theorem 3.7 and Corollary 3.8, we obtain

$$
\begin{aligned}
& \mathcal{T}_{Y}=\left\{\emptyset, Y, \cup_{i \in I} R\left[x_{i}\right] \mid I \subset\{1,2, \ldots, 6\}\right\}, \\
& \mathcal{F}_{Y}=\left\{\emptyset, Y, \cap_{i \in I} R^{c}\left[x_{i}\right] \mid I \subset\{1,2, \ldots, 6\}\right\}, \\
& \mathcal{T}_{X}=\left\{\emptyset, X, \cup_{i \in I} R^{-1}\left[y_{i}\right] \mid I \subset\{1,2, \ldots, 5\}\right\}, \\
& \mathcal{F}_{X}=\left\{\emptyset, X, \cap_{i \in I} R^{-1 c}\left[y_{i}\right] \mid I \subset\{1,2, \ldots, 5\}\right\} .
\end{aligned}
$$

\section{References}

[1] R. Bělohlávek, Fuzzy Relational Systems, Kluwer Academic Publishers, New York, 2002.

[2] D.G. Chen, J.Q. Liu, E.C.C. Tsang, Granular computing based on fuzzy similarity relations, Soft Computing, 15(2010) 1161-1172.

[3] J. Dai, H. Han, X.Zhang, M. Liu, S. Wan, J.Liu, Z. Lu, Catoptrical rough set model on two universes using granule-based definition and its variabble precision extesions, article in press Information Sciences.

[4] D. Dubois, H. Prade, Rough fuzzy sets and fuzzy rough sets, Int. J. Gen. Syst., 17(1990) 191-209. 
[5] B.Q. Hu, H. Wong, Generalized interval-valued fuzzy variable precision rough set, Int.J. Fuzzy Sets Syst., 16(2014) 554-565.

[6] J. Kortelainen, On relationships between modified sets, topological spaces and rough sets, Fuzzy Sets and Systems, 61(1994) 91-95.

[7] Z. Pawlak, Rough sets, Int. J. Comput. Inf. Sci., 11 (1982) 341-356.

[8] Z. Pawlak, Rough probability, Bull. Pol. Acad. Sci. Math., 32(1984) 607-615.

[9] J. Qiao, B.Q. Hu, Granular variable precision $L$-fuzzy rough sets based on residuated lattices, article in press Fuzzy Sets and Systems

[10] C.Y. Wang, B.Q. Hu, Granular variable precision fuzzy rough sets with general fuzzy relations Fuzzy Sets and Systems, 275 (2015) 39-57, doi: 10.1016/j.fss.2015.01.016.

[11] W.Z.Wu, J.S.Mi, W.X.Zhang, Generalized fuzzy rough sets, Information Sciences, 151(2003) 262-282, doi: 10.1016/SOO20-0255(02)00379-1.

[12] W.Z.Wu, Y. Leung, J.S. Mi, On characterizations of $(\varphi, \tau)$-fuzzy rough approimation operators, Fuzzy Sets and Systems, 154 (2005) 76-102, doi: 10.1016/j.fss.2005.02.011.

[13] Y. Yao, J.Mi, Z. Li, A novel variable preision $(\theta, \sigma)$-fuzzy rough set model based on fuzzy granules, Fuzzy Sets and Systems, 236 (2014) 58-72, doi: 10.1016/j.fss.2013.06.012.

[14] Y.Y. Yao, Constructive and algebraic methods of theory of rough sets, Information Sciences, 109 (1998), 21-47.

[15] Y.Y. Yao, Three-way decisions with probabilitistic rough sets, Information Sciences, 180 (2010), 341-353,doi: 10.1016/j.ins.2009.09.021.

[16] Zhen Ming Ma, Bao Qing Hu, Topological and lattice structures of L-fuzzy rough set determined by lower and upper sets, Information Sciences, 218 (2013), 194-204,doi: 10.1016/j.ins.2012.06.029.

[17] W. Ziarko, Variable precision rough set model, Journal of Computer and System Sciences, 46 (1993) 39-59. 
